\title{
Direct Electric-Field Induced Phase Transformation in Paraelectric Zirconia via Electrical Susceptibility Mismatch
}

\author{
Alan Lai and Christopher A. Schuh $\odot^{*}$ \\ Department of Materials Science and Engineering, Massachusetts Institute of Technology, \\ Cambridge, Massachusetts 02139, USA
}

(Received 20 August 2020; accepted 8 December 2020; published 6 January 2021)

\begin{abstract}
Electric field driven phase transformations require two phases with a mismatch in their electric polarization, as seen in antiferroelectric-to-ferroelectric transformations, where the ferroelectric phase has a permanent polarization that is favored under field. Many other nonferroelectric dielectric materials can become electrically polarized according to their electrical susceptibility, yet such induced polarizations are not generally considered capable of enabling a phase transformation. Here we explore a susceptibilitymismatch phase transformation in a paraelectric ceramic, yttria-doped zirconia. Using in situ x-ray diffraction at $550{ }^{\circ} \mathrm{C}$ we show that the monoclinic-to-tetragonal transformation can be driven directly by an electric field, providing experimental evidence of a paraelectric-to-paraelectric phase transformation. Considering the $\sim 1 \%$ mechanical strain of this transformation, the resulting electromechanical coupling may have potential for solid-state electrical actuators.
\end{abstract}

DOI: 10.1103/PhysRevLett.126.015701

The most common materials that transduce electrical energy into mechanical strain are ferroelectrics and piezoelectrics [1-6], and they achieve this coupling with special crystal structures that are noncentrosymmetric [7-9]. Unfortunately, this crystallographic requirement puts a significant limitation on the number of candidate materials with electroactive properties. A related way to generate large strains is through electrically triggering a solid-state phase transformation [10], where the critical ingredient is a material property difference between the two phases. This can be seen in antiferroelectric-to-ferroelectric transformations that are induced by an electric field [11] where the spontaneous polarization of the ferroelectric phase is much greater than the antiferroelectric phase. This is illustrated schematically in the polarization vs field diagram shown in Fig. 1(a), where only the ferroelectric phase has a net spontaneous polarization and as the field is increased there is a rapid jump in polarization upon phase transformation that is recovered on the reverse transformation (albeit with some hysteresis).

Static polarization differences are often so large in magnitude that they are taken to be the only contribution to driving the transformation. However, there are higherorder material properties that contribute to the overall polarization [12-14] revealed by the Taylor expansion,

Published by the American Physical Society under the terms of the Creative Commons Attribution 4.0 International license. Further distribution of this work must maintain attribution to the author(s) and the published article's title, journal citation, and DOI.

$$
\boldsymbol{P}(E)=\boldsymbol{P}(0)+\frac{d \boldsymbol{P}(0)}{d \boldsymbol{E}} \boldsymbol{E}+\frac{d^{2} \boldsymbol{P}(0)}{d \boldsymbol{E}^{2}} \boldsymbol{E}^{2}+\cdots,
$$

where the bolded terms $\boldsymbol{P}$ and $\boldsymbol{E}$ are tensor quantities representing the polarization and electric field, respectively, and the derivatives represent coefficients, or materials properties, that affect polarization. For example, $d \boldsymbol{P} / d \boldsymbol{E}$ comprises the well-known electrical susceptibility, $\chi$ [8], and if the series is truncated at the linear term we obtain,

$$
P(E) \approx \boldsymbol{P}^{s p}+\varepsilon_{0} \chi \boldsymbol{E},
$$

where the first term, $\boldsymbol{P}^{s p}=\boldsymbol{P}(0)$, is the spontaneous polarization at zero field, and $\varepsilon_{0}$ is the permittivity of free space [8].

To examine the transformation between two phases we write a thermodynamic potential $d \Psi=-S \cdot d T+P \cdot d E$, with $S$ the entropy and $T$ the temperature, and perform a Clausius-Clapeyron analysis [15] that equates the potential of the two phases at their equilibrium transformation temperature, $T_{0}$.

$$
\frac{d \boldsymbol{E}}{d T}=-\frac{\Delta S}{\left(\Delta \boldsymbol{P}^{s p}+\varepsilon_{0} \cdot \chi \boldsymbol{E}\right)} .
$$

Here the delta terms represent the difference in material properties between the phases; these are essential for the transformation to occur under an electric field. A full derivation of Eq. (3) is provided in the Supplemental Material [16]. One way to interpret Eq. (3) is that the denominator is the electrical work put into the system, 


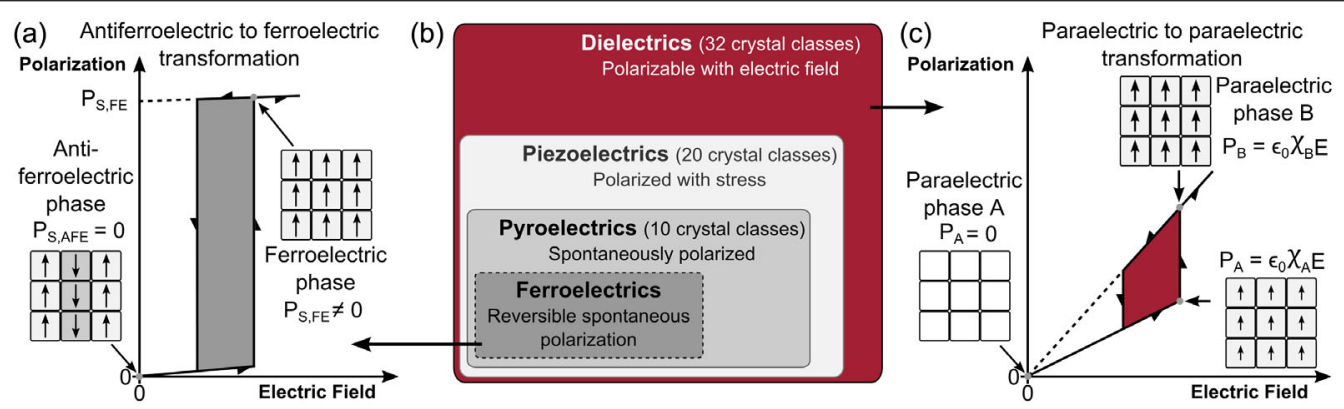

FIG. 1. Comparison of electric field induced transformations between phase $A$ and $B$ in electric polarization-field space. In an antiferroelectric-to-ferroelectric transformation (a) there is only a difference in the spontaneous polarization between the two phases, which is given by the phases' $y$ intercepts. (b) Expanding beyond the small subset of ferroelectric systems that make up the whole domain of dielectric materials [7] increases the range of available materials. In a paraelectric-to-paraelectric transformation (c) the polarization difference arises from the electrical susceptibility of each phase, given by the different slopes of the two phases.

which must exceed the entropy term in the numerator for the transformation to proceed.

Importantly, Eq. (3) also indicates that a phase transformation can be enabled by either a difference in the spontaneous polarization $\left(\Delta \boldsymbol{P}^{s p}\right)$ or a susceptibility mismatch $(\Delta \chi)$. This latter point is unusual, because in typical electrically driven phase transformations, $\Delta \boldsymbol{P}^{s p}$ is vastly larger than $\Delta \chi$ [11], and as a result the susceptibility is usually neglected. However, we propose that in certain systems the situation could be reversed, with $\Delta \boldsymbol{P}^{s p}$ negligible compared to $\Delta \chi$. For example, paraelectric materials by definition have no spontaneous polarization but can be polarized with applied electric fields; in these materials it is possible that a susceptibility mismatch can become the dominant contributor that drives a phase transformation. This concept is schematized in Fig. 1(c), where a paraelectric material becomes polarized and once it reaches a large enough field energy input it transforms to another paraelectric phase with a larger susceptibility. While the physical outcomes of the two situations in Figs. 1(a) and 1(c) are similar, they are fundamentally differentiated by the relative dominance of the spontaneous and susceptibility terms in Eq. (3).

Here we study the susceptibility-driven martensitic phase transformation in bulk single-crystal zirconia $[17,18]$ from its low temperature monoclinic phase to its high temperature tetragonal phase, which is usually triggered by heat or stress. For bulk zirconia we expect the transformation to be susceptibility driven because it is definitively not ferroelectric or piezoelectric: the phases involved are centrosymmetric (tetragonal- $P 4_{2} / n m c$, and monoclinic- $P 2_{1} / c$ ) [17]. While there is literature reporting that $\mathrm{HfO}_{2}$ and $\mathrm{ZrO}_{2}$ thin films can exhibit ferroelectricity when they are distorted by epitaxial stresses into an orthorhombic (ferroelectric) phase, this is limited to highly constrained epitaxial films with thicknesses of about $10 \mathrm{~nm}$ [19-23] and the ferroelectric properties tend to diminish as the film thickness is increased. But for bulk zirconia ( $\sim 100 \mu \mathrm{m}$ thick) with no epitaxial (or other) constraints as in our present work, the only relevant phases are the monoclinic and tetragonal phases [24]. These phases have a difference in the electrical susceptibility of $\sim 18$ [25] (see Supplemental Material [16]), which amounts to slopes in Fig. 1(c) that are different by more than a factor of 2 . In what follows we will show that this is large enough that an electric field can drive the transformation, from the monoclinic phase to the more polarizable tetragonal phase according to Eq. (3).

Table S1 presents the full specimen preparation regimen for this work, which begins with $\sim 3 \times 3 \times 3 \mathrm{~mm}$ crystals of $2.0 \mathrm{~mol} \% \mathrm{Y}_{2} \mathrm{O}_{3}-\mathrm{ZrO}_{2}$ produced via cold-crucible induction melting [26]. These were ground to produce two parallel faces with a thickness of about 1-2 $\mathrm{mm}$ and a roughly equiaxed (but irregular) shape in the plane. Transformation temperatures were measured using differential scanning calorimetry (DSC), see Fig. S1 and Table S2. Test specimens were further processed using a disc polisher to a final thickness of about $125 \mu \mathrm{m}$. Both sides were coated with metallic electrodes $\sim 125 \mathrm{~nm}$ thick - thin enough to permit $\mathrm{x}$-ray transmission. The sample was then mounted with silver paste onto platinum foil atop fused silica and copper wire leads were attached to the electrodes with silver paste. The experimental structure, design, and processing are given in the Supplemental Material [16].

General area x-ray diffraction (GAXDS) was used to identify the angular positioning for each diffraction peak. A high resolution $\mathrm{x}$-ray diffraction point detector equipped with a heating stage was then focused on the relevant peaks, and rocking curve scans were collected around the key peaks during heating and cooling, and before and after the application of an electric field; see Supplemental Material [16].

Figure 2 shows rocking curve data that track the magnitudes of the dominant peaks of the two phases (monoclinic 111 reflection at $2 \theta=31^{\circ}$ and tetragonal 111 reflection at $2 \theta=30$ ) during our experiment, and the integrated areas under those peaks. The breadth of the peaks could be attributed to, e.g., chemical inhomogeneity 


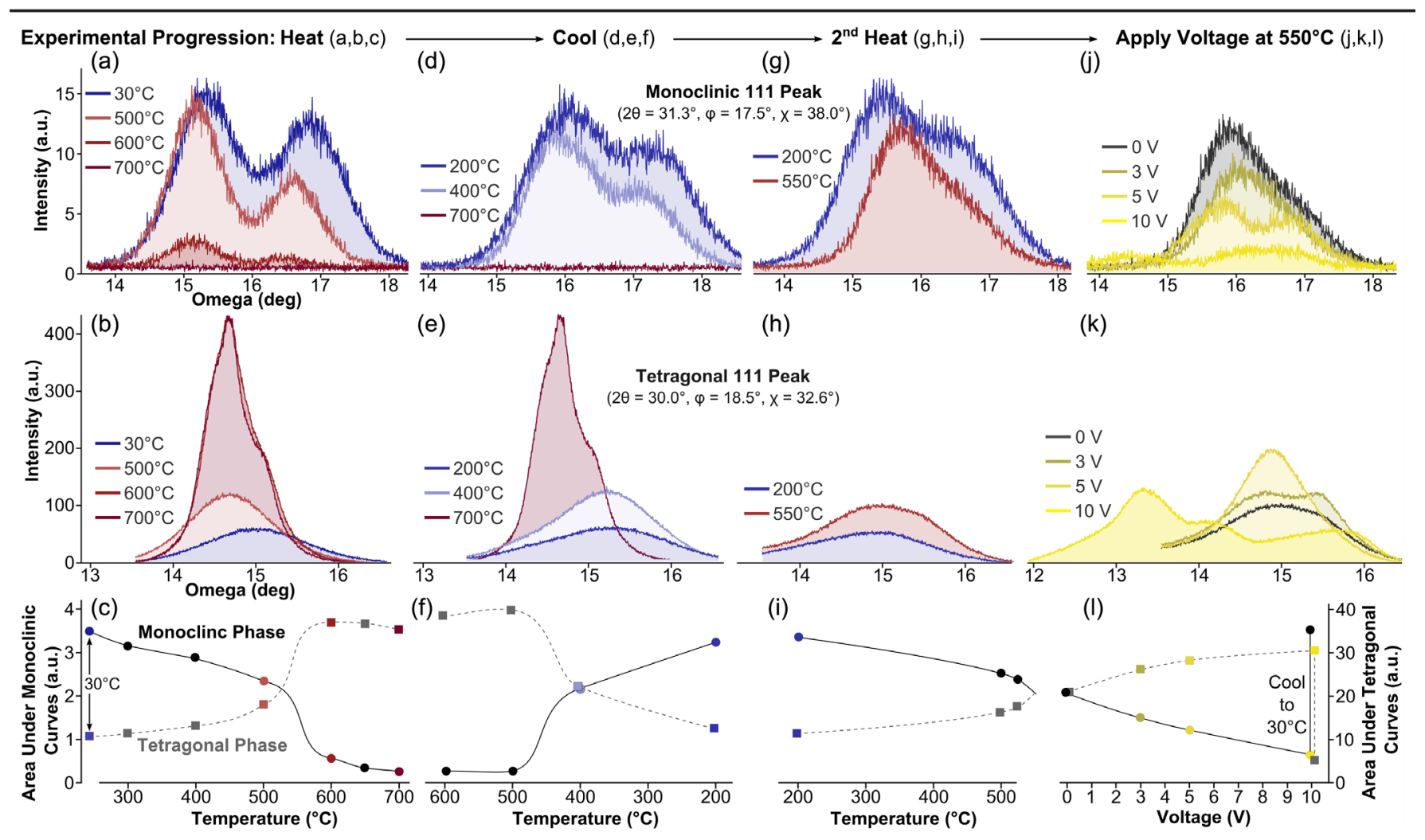

FIG. 2. In situ XRD rocking curves showing the martensitic transformation in zirconia triggered with heat and with field. Rocking curves for sample 1, using arbitrary units (a.u.), for the monoclinic (top row) and tetragonal (middle row) phases. The monoclinic peaks shrank upon heating to $700{ }^{\circ} \mathrm{C}$ (a) with concurrent growth of the tetragonal peaks (b). Cooling to $200^{\circ} \mathrm{C}$ (d), (e) recovered most of the transformation. Samples were reheated to $550^{\circ} \mathrm{C}(\mathrm{g}),(\mathrm{h})$ before a voltage was applied (j), (k), causing the monoclinic peaks to shrink and tetragonal ones to grow in a similar manner to the thermal heating transformation. The corresponding integrated areas under the curves are shown in the bottom row, and trace the waxing and waning of the phases through these cycles.

or strain variations but we have no reason to expect chemical inhomogeneity in skull melted crystals processed in the liquid state [27]. We therefore expect that the peak breadth is likely reflective of a strained condition due to variant competition and partial transformation. As the transformation proceeds, we see changes in the shape and width of the peaks, occasionally including splitting into superimposed pairs. These rocking curves thus certainly sample a complex structure of variant assemblages, strain distributions [28], etc., which evolve throughout the transformation. It is difficult to quantify the phase fraction by comparing peak intensities since only a few reflections can be targeted at a time in these in situ experiments. However, the presence of the two phases is roughly proportional to the integrated intensities of the rocking curve peaks, and indicates that the crystals are majority in the tetragonal phase at room temperature.

Upon heating to $700{ }^{\circ} \mathrm{C}$, the monoclinic peaks decreased and eventually disappeared, Fig. 2(a), replaced by tetragonal peaks starting around $450{ }^{\circ} \mathrm{C}$, Fig. 2 (b), as the sample transforms. Upon cooling from $700^{\circ} \mathrm{C}$ the reverse effect was observed, with phase reversion (and expected hysteresis) beginning around $500{ }^{\circ} \mathrm{C}$ and completing near $300^{\circ} \mathrm{C}$ Figs. 2(d) $-2(\mathrm{f})$.

After this thermal cycle, sample 1 was heated again up to $550^{\circ} \mathrm{C}$, which is approximately $50^{\circ}$ above where the thermal transformation to the tetragonal phase begins, (but well below the temperature where it completes $\sim 625^{\circ} \mathrm{C}$ ). This led to small changes to both sets of peaks due to the partial transformation, as shown by the "second heating" peaks in Fig. 2(g) and 2(h). After equilibrating at $550^{\circ} \mathrm{C}$, a dc voltage was applied for approximately $1 \mathrm{sec}$. Rocking curves were subsequently collected with the voltage turned off. Figures 2(j) and 2(k) show how each peak responded to the voltage, with the monoclinic peak decreasing and the tetragonal peak growing as the voltage increased. At $10 \mathrm{~V}(0.9 \mathrm{kV} / \mathrm{cm})$, the monoclinic peak nearly disappeared, indicating the voltage drove the transformation to completion in a manner similar to how an increase in temperature would. The close similarity of the electrically and thermally triggered peak changes [compare Figs. 2(a) and 2(j), as well as Figs. 2(b) and 2(k)], which in turn are supported by complementary DSC and TMA (Supplemental Material [16]), strongly suggests that phase transformation is occurring in these experiments. 
The post-voltage peaks exhibited a shift in omega angle by about $2^{\circ}$. This is attributed to an out-of-plane displacement due to a portion of the film detaching from the substrate (likely due to the transformation strain), and persists for all the subsequent experiments. After cooling to room temperature the peaks are essentially fully recovered (Fig. S6 A,B) indicating the electric field-driven transformation can be reversed thermally. This result is reproducible: a similar experiment was performed on a second, separate crystal with similar results shown in Figs. S4 and S5.

Figure 3 summarizes the response of each phase to the stimuli and shows that the phase transformation can be driven by either temperature or voltage. While zirconia can become ionically conductive due to oxygen vacancy mobility at elevated temperatures [29], we do not expect that such effects aid this transformation, as it is a diffusionless martensitic transformation. What is more, we do not suspect electrical heating, as the conductivity of zirconia [29-32] is too low to sustain even one degree of Joule heating over the voltage application time of $\sim 1 \mathrm{sec}$ used here (see Supplemental Material [16]).

We propose that the paraelectric-to-paraelectric transformation seen in zirconia here is driven in the manner of Fig. 1(c), by the difference in the electrical susceptibility. We expand upon Eq. (3) by relating the transformation entropy to the enthalpy of transformation $(\Delta H)$ as $\Delta S=\Delta H / T_{0}$,

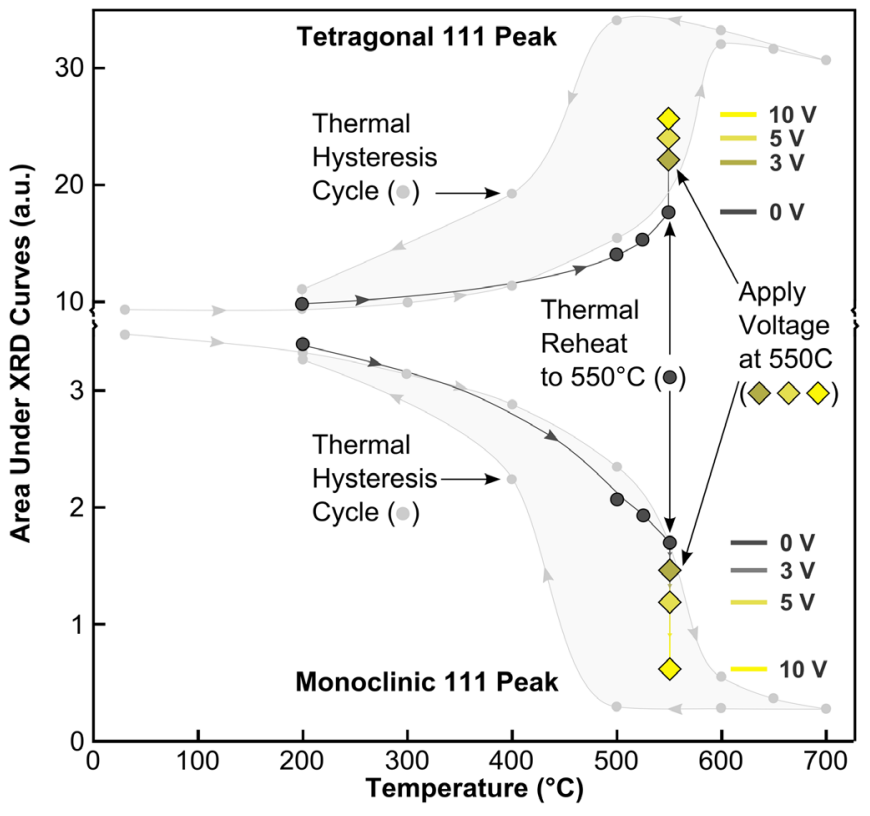

FIG. 3. Electric field induced transformation overlaid on the thermal transformation to illustrate their similarity. The integrated areas under XRD rocking curves (such as those in Fig. 2) are plotted against temperature for a thermal cycle (light gray circles), reheating to $550{ }^{\circ} \mathrm{C}$ (dark gray circles), and finally application of voltage which produces the phase transformation from monoclinic to tetragonal as indicated by the gray-to-yellow diamonds. introducing Einstein notation (see Supplemental Material [16]) for a full derivation), and integrating to yield the electric field required to drive the transformation as,

$\Delta H\left(1-\frac{T}{T_{0}}\right)=\Delta \boldsymbol{P}_{i}^{s p} \boldsymbol{E}_{i}+\varepsilon_{0} \Delta \chi_{i j} \boldsymbol{E}_{j} \boldsymbol{E}_{i}-\frac{1}{2} \varepsilon_{0} \Delta \chi_{i i} \boldsymbol{E}_{i} \boldsymbol{E}_{i}$.

Equation (4) contains many anisotropic terms but if, for simplicity, we assume the applied field is uniaxial and the materials properties are isotropic then it takes a quadratic form that can be explicitly solved to yield the form of the $E-T$ phase boundary,

$$
E=\frac{-\Delta P^{s p} \pm \sqrt{\left(\Delta P^{s p}\right)^{2}+2 \varepsilon_{0} \Delta \chi \Delta H\left(1-\frac{T}{T_{0}}\right)}}{\varepsilon_{0} \Delta \chi},
$$

This result reduces to that of Yang and Payne [11] for the antiferroelectric-to-ferroelectric transformation in the limiting case where the susceptibility is assumed negligible $(\Delta \chi \rightarrow 0)$ :

$$
E_{\Delta \chi \rightarrow 0}= \pm \frac{\Delta H\left(1-\frac{T}{T_{0}}\right)}{\Delta P^{s p}}
$$

In the present experiments, we are operating in the complementary limit, where there is no spontaneous polarization difference between the two phases $\left(\Delta P^{s p}=0\right)$ so the only contribution comes from the susceptibility difference ( $\Delta \chi \sim 18$ for isotropic zirconia) for which Eq. (5) reduces to

$$
E_{\Delta P \rightarrow 0}= \pm \sqrt{\frac{2 \Delta H\left(1-\frac{T}{T_{0}}\right)}{\varepsilon_{0} \Delta \chi}} .
$$

When Eqs. (6) and (7) are schematically plotted to create a phase diagram (taking $\Delta H>0, \Delta P>0$ and $\Delta \chi>0$ here), they show entirely different forms; for transformations with only spontaneous polarization [Fig. 4(a)], the phase boundary is linear, but for solely paraelectric systems [Fig. 4(b)] the relationship is parabolic.

The maximum electric field that can be applied to a material is given by the point of dielectric breakdown, which is illustrated in Fig. 4 with a horizontal dashed line. This limit, along with the theoretical phase boundary [Eqs. (6) or (7)], defines a region where the electric field induced transformation is accessible, indicated by the darkly shaded regions. In the purely paraelectric transformation, Fig. 4(b), the intersection of these two curves also defines the lowest temperature at which we can hope to trigger the transformation with an applied field, and is found by substituting the breakdown field $\left(E_{\mathrm{BD}}\right)$ into Eq. (7). Using literature values for $\Delta H=2.0 \mathrm{~kJ} / \mathrm{mol}$ [33] and $\Delta \chi=18 \quad[13]$ and $E_{\mathrm{BD}}=5 \mathrm{MV} / \mathrm{cm}$ [34-37] (see Supplemental Material [16]) for details), this analysis 


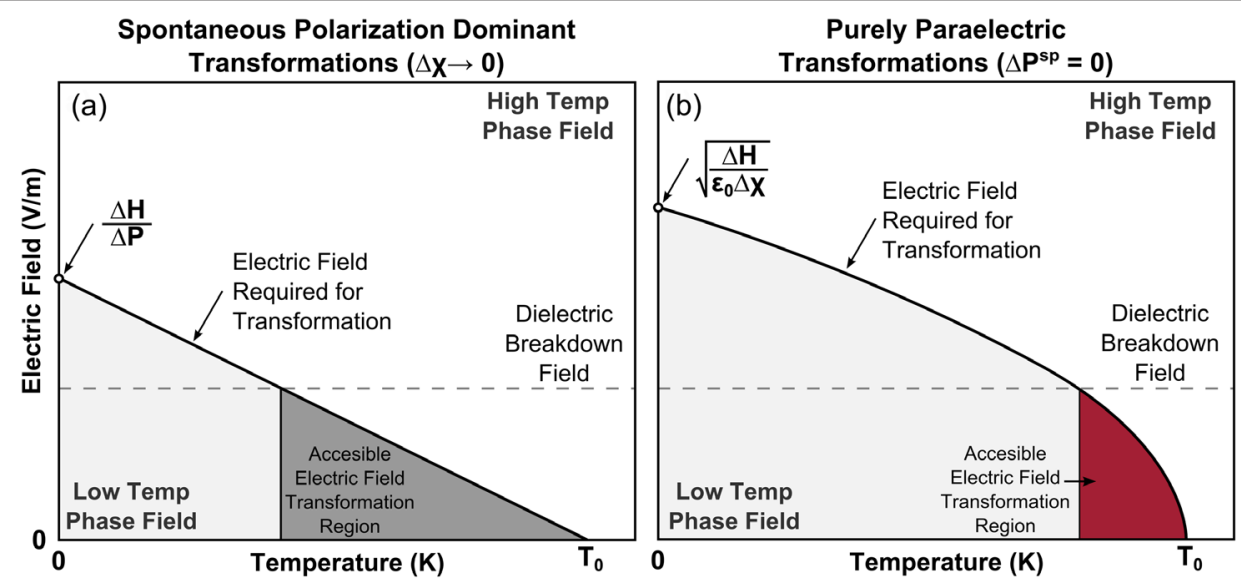

FIG. 4. Schematic electric field-temperature phase diagrams for electric field induced phase transformations. (a) System that is dominated by the spontaneous polarization of one or more of the phases and shows classic, linear Clausius-Clapeyron response. (b) System that is purely paraelectric with neither phase having any spontaneous polarization, the field required for the transformation is nonlinear and governed by Eq. (7). The dielectric breakdown field (dashed line) is assumed to be independent of temperature.

suggests that temperatures over $\sim 400^{\circ} \mathrm{C}$ are required to see this effect, and the width of the accessible region is on the order of $150^{\circ} \mathrm{C}$. The limited width of this region may help explain why susceptibility mismatch transformations have not been previously explored in this manner; it would be a rare condition to encounter fortuitously, without intentionally targeting it as we have.

It is also notable that the transformation did not reverse when the electric field was removed, which is in contrast to what might be expected from the distinct regions of the phase diagram in Fig. 4. This is explained by the hysteretic (path dependent) transformation behavior of zirconia, where in the range of $\sim 350-650^{\circ} \mathrm{C}$ either phase could be dominant depending on the thermal history. However, the theory does suggest that at certain temperatures the transformation could be fully reversible with field only, in a manner analogous to superelasticity in stress-induced martensitic transformations [15]; this is an interesting direction for future work.

Lastly, this field-driven transformation is also one that leads to mechanical actuation, as the transformation strains in zirconia are $\sim 16 \%$ in shear [17], and can amount to as much as 7\% linear strain in the best orientations [38]. Here we separately measured the transformation strain as $1 \%$ (Fig. S7); using a thermal dilatometer on our second sample before thinning while the crystal was $1 \mathrm{~mm}$ thick (as described in the Supplemental Material [16]). We then thinned and mounted the crystal and demonstrated that we could activate the transformation with voltage (Figs. S4 and S5). Together, these results show that the electric field induced transformation should produce $\sim 1 \%$ strain (or more with some orientation optimization as noted above), which could be potentially significant in the landscape of electroactive ceramics, the best of which currently achieve $\sim 0.8 \%$ strain [39].

Finally, we observe that the concept of using higher order material properties differences to drive phase transformations could extend to other electrical properties not yet explored in this context, such as pyroelectric or piezoelectric coefficients (see Supplemental Material [16]). There may also be interesting analogies with other higherorder material properties in mechanical or magnetic systems [40-46],

and their ability to drive phase transformations should be explored. We expect that the ability to trigger phase transformations using solely susceptibility differences shown here should extend the range of electroactive ceramics that can be studied. Many dielectrics that might normally be ignored as functional materials due to their lack of a spontaneous polarization may in fact be active paraelectric materials by means of a susceptibility mismatch transformation.

The authors acknowledge the helpful conversations with Professor E. Homer and Professor O. Johnson (BYU) and Professor J. LeBeau, Professor W. C. Carter, E. Pang, I. Crystal, Dr. M. Gibson, Dr. Z. Cordero, Dr. C. Settens, and $\mathrm{K}$. Broderick (MIT). Kind financial support from the Deshpande Center at MIT, the Kavanaugh Fellows Program in the Department of Materials Science and Engineering at MIT, and the Institute for Soldier Nanotechnologies funded by the U.S. Army Research Office under Collaborative Agreement No. W911NF-182-0048. This work employed MIT MRL XRD Shared Experimental Facilities supported in part by the MRSEC Program of the NSF under Grant No. DMR 1419807.

*Corresponding author schuh@mit.edu

[1] X. Ren, Nat. Mater. 3, 91 (2004).

[2] J. X. Zhang, B. Xiang, Q. He, J. Seidel, R. J. Zeches, P. Yu, S. Y. Yang, C. H. Wang, Y.-H. Chu, L. W. Martin, M. A. M., and R. Ramesh, Nat. Nanotechnol. 6, 98 (2011).

[3] S. H. Baek et al., Science 334, 958 (2011). 
[4] C. Hu, X. Meng, M. Zhang, H. Tian, J. E. Daniels, P. Tan, F. Huang, L. Li, K. Wang, J. Li, Q. Lu, W. Cao, and Z. Zhou, Sci. Adv. 6, 1 (2020).

[5] H. He and X. Tan, Phys. Rev. B 72, 024102 (2005).

[6] P. Muralt, R. G. Polcawich, and S. Trolier-McKinstry, MRS Bull. 34, 658 (2009).

[7] G. H. Haertling, J. Am. Ceram. Soc. 82, 797 (1999).

[8] D. Damjanovic, Rep. Prog. Phys. 61, 1267 (1998).

[9] F. Jin, L. Wang, A. Zhang, J. Ji, Y. Shi, X. Wang, R. Yu, J. Zhang, E. W. Plummer, and Q. Zhang, Proc. Natl. Acad. Sci. U.S.A. 116, 20322 (2019).

[10] C. C. Koch, Mater. Sci. Eng. A 287, 213 (2000).

[11] P. Yang and D. A. Payne, J. Appl. Phys. 71, 1361 (1992).

[12] J. P. Locquet, C. Marchiori, M. Sousa, J. Fompeyrine, and J. W. Seo, J. Appl. Phys. 100, 051610 (2006).

[13] D. P. Thompson, A. M. Dickins, and J. S. Thorp, J. Mater. Sci. 27, 2267 (1992).

[14] A. I. Kingon, J.-P. Maria, and S. K. Streiffer, Nature (London) 406, 1032 (2000).

[15] K. Otsuka and K. Shimizu, Int. Mater. Rev. 31, 93 (1986).

[16] See Supplemental Material at http://link.aps.org/ supplemental/10.1103/PhysRevLett.126.015701 for susceptibility tensors of zirconia, experimental setup and $\mathrm{x}$-ray diffraction details, material properties of zirconia, resistive heating calculations, full thermodynamic derivation, and mechanical strain measurements.

[17] R. H. J. Hannink, P. M. Kelly, and B. C. Muddle, J. Am. Ceram. Soc. 83, 461 (2000).

[18] J. Chevalier, L. Gremillard, A. V. Virkar, and D. R. Clarke, J. Am. Ceram. Soc. 92, 1901 (2009).

[19] M. H. Park, Y. H. Lee, H. J. Kim, Y. J. Kim, T. Moon, K. Do Kim, J. Müller, A. Kersch, U. Schroeder, T. Mikolajick, and C. S. Hwang, Adv. Mater. 27, 1811 (2015).

[20] J. Müller, T. S. Böscke, U. Schröder, S. Mueller, D. Bräuhaus, U. Böttger, L. Frey, and T. Mikolajick, Nano Lett. 12, 4318 (2012).

[21] J. Müller, T. S. Böscke, D. Bräuhaus, U. Schröder, U. Böttger, J. Sundqvist, P. Kcher, T. Mikolajick, and L. Frey, Appl. Phys. Lett. 99, 112901 (2011).

[22] B. T. Lin, Y. W. Lu, J. Shieh, and M. J. Chen, J. Eur. Ceram. Soc. 37, 1135 (2017).

[23] R. Materlik, C. Kunneth, and A. Kersch, J. Appl. Phys. 117, 134109 (2015).

[24] E. H. Kisi and C. J. Howard, Key Eng. Mater. 153-154, 1 (1998).

[25] X. Zhao and D. Vanderbilt, Phys. Rev. B 65, 075105 (2002).
[26] V. I. Aleksandrov, V. V. Osikov, A. M. Prokhoros, and V. M. Tatarintsev, Russ. Chem. Rev. 47, 213 (1978).

[27] M. A. Borik, E. E. Lomonova, V. V. Osiko, V. A. Panov, O. E. Porodinkov, M. A. Vishnyakova, Y. K. Voron'Ko, and V. V. Voronov, J. Cryst. Growth 275, e2173 (2005).

[28] G. Arlt and P. Sasko, J. Appl. Phys. 51, 4956 (1980).

[29] V. V. Kharton, F. M. B. Marques, and A. Atkinson, Solid State Ionics 174, 135 (2004).

[30] E. N. S. Muccillo and M. Kleitz, J. Eur. Ceram. Soc. 16, 453 (1996).

[31] O. H. Kwon, C. Jang, J. Lee, H. Y. Jeong, Y. il Kwon, J. H. Joo, and H. Kim, Ceram. Int. 43, 8236 (2017).

[32] T. K. Gupta, R. B. Grekila, and E. C. Subbarao, J. Electrochem. Soc. 128, 929 (1981).

[33] A. Suresh, M. J. Mayo, and W. D. Porter, J. Mater. Res. 18, 2912 (2003).

[34] E. S. Ramakrishnan, K. D. Cornell, G. H. Shapiro, and W.-Y. Howng, J. Electrochem. Soc. 145, 358 (1998).

[35] O. Jongprateep, V. Petrovsky, and F. Dogan, J. Met. Mater. Miner. 18, 9 (2008).

[36] K. Kukli, K. Forsgren, M. Ritala, M. Leskela, J. Aarik, and A. Hårsta, J. Electrochem. Soc. 148, F227 (2001).

[37] D. H. Kuo, C. H. Chien, and C. H. Huang, Thin Solid Films 420-421, 47 (2002).

[38] X. M. Zeng, A. Lai, C. L. Gan, and C. A. Schuh, Acta Mater. 116, 124 (2016).

[39] C. Chen, X. Zhao, Y. Wang, H. Zhang, H. Deng, X. Li, X. Jiang, X. Jiang, and H. Luo, Appl. Phys. Lett. 108, 1 (2016).

[40] T. Omori, K. Watanabe, R. Y. Umetsu, R. Kainuma, and K. Ishida, Appl. Phys. Lett. 95, 082508 (2009).

[41] Y. Tanaka, Y. Himuro, R. Kainuma, Y. Sutou, T. Omori, and K. Ishida, Science 327, 1488 (2010).

[42] R. S. McWilliams, D. K. Spaulding, J. H. Eggert, P. M. Celliers, D. G. Hicks, R. F. Smith, G. W. Collins, and R. Jeanloz, Science 338, 1330 (2012).

[43] Y. Gao, X. Wang, L. Xie, Z. Hu, H. Lin, Z. Zhou, T. Nan, X. Yang, B. M. Howe, J. G. Jones, G. J. Brown, and N. X. Sun, Appl. Phys. Lett. 108, 232903 (2016).

[44] S. Rößler, C. Koz, Z. Wang, Y. Skourski, M. Doerr, D. Kasinathan, H. Rosner, M. Schmidt, U. Schwarz, U. K. Rößler, and S. Wirth, Proc. Natl. Acad. Sci. U.S.A. 116, 16697 (2019).

[45] W. Kriven, J. Am. Ceram. Soc. 71, 1021 (1988).

[46] A. Pramanick, A. Glavic, G. Samolyuk, A. A. Aczel, V. Lauter, H. Ambaye, Z. Gai, J. Ma, A. D. Stoica, G. M. Stocks, S. Wimmer, S. M. Shapiro, and X. L. Wang, Phys. Rev. B 92, 134109 (2015). 